Elycée TINDANO ${ }^{1}$

Souleymane GANABA ${ }^{2}$

Oumarou SAMBARE ${ }^{3}$

Adjima THIOMBIANO ${ }^{1}$

${ }^{1}$ Université de Ouagadougou UFR/SVT, Laboratoire de

biologie et écologie végétales BP 7021, Ouagadougou 03

Burkina Faso

${ }^{2}$ Institut de l'environnement et de recherches agricoles (INERA) CREAF de Kamboinsé Département Productions forestières

BP 7047, Ouagadougou 03

Burkina Faso

3 Institut des Sciences (IDS) BP 1757, Ouagadougou 03

Burkina Faso

\title{
La végétation des inselbergs du Sahel burkinabè
}

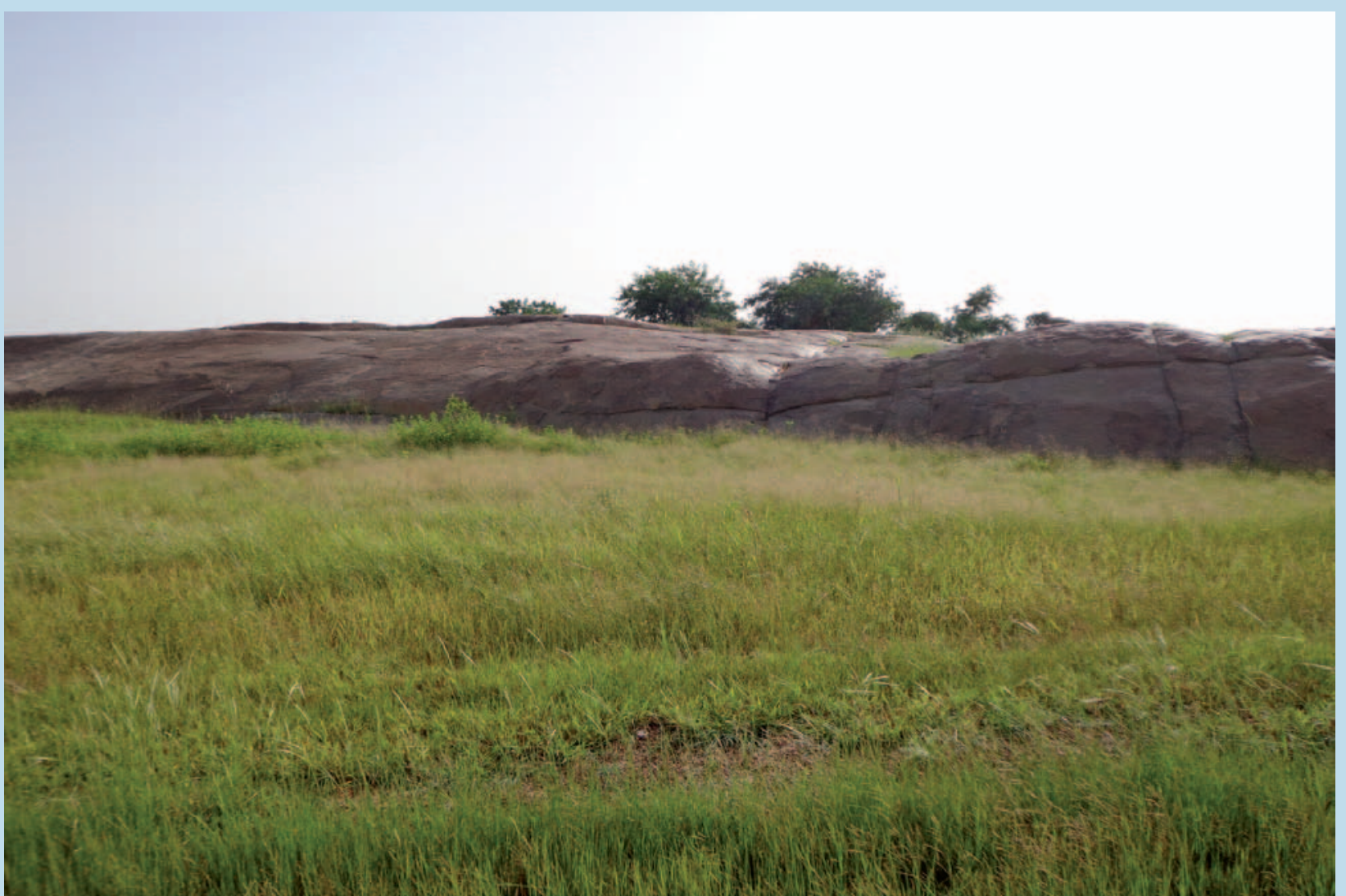

Photo 1.

Inselberg granitique.

Photo E. Tindano, Sampelga. 


\section{RÉSUMÉ}

\section{LA VÉGÉTATION DES INSELBERGS DU SAHEL BURKINABÈ}

Les inselbergs sont des affleurements rocheux caractérisés par la précarité de leurs facteurs édaphiques et microclimatiques. L'objectif de cette étude est de faire une étude comparative entre la végétation des inselbergs et celle des milieux environnants. Les données ont été collectées dans des placeaux de $900 \mathrm{~m}^{2}$ pour la strate ligneuse et de $100 \mathrm{~m}^{2}$ pour la strate herbacée suivant un échantillonnage stratifié et aléatoire. Un total de 162 relevés a été réalisé. L'étude a permis d'inventorier 228 espèces végétales réparties dans 43 familles et 144 genres. Sur les inselbergs, 184 espèces ont été recensées et sont réparties dans 136 genres et 36 familles. Seule l'espèce Cynanchum hastifolium a été recensée comme endémique stricte sur les inselbergs. Dans les milieux environnants des inselbergs, le cortège floristique est composé de 194 espèces réparties dans 40 familles et 127 genres. Le nombre moyen d'espèces par relevé est plus élevé dans les milieux environnants $(23,53 \pm 6,28)$ que dans les inselbergs $(19,93 \pm 4,83)$. La végétation des inselbergs (719,47 $\pm 337,27$ pieds/ ha) est moins dense que celle des milieux environnants ( $1389,91 \pm 674,79$ pieds/ ha). D'une part, l'impact anthropique plus prononcé dans les plaines environnantes et, d'autre part, les espèces reliques et la dominance de Pterocarpus lucens sur les inselbergs traduiraient le caractère refuge des inselbergs pour certaines espèces végétales. La différence entre la végétation des inselbergs et celle des milieux environnants est liée aux facteurs édaphiques et anthropiques.

Mots-clés : inselbergs, phytosociologie, écologie, Burkina Faso.

\section{ABSTRACT}

\section{SAHELIAN INSELBERG VEGETATION IN BURKINA FASO}

Inselbergs are rocky outcrops characterised by the precarious nature of their edaphic and micro-climatic factors. The aim of this study was to compare the vegetation on inselbergs with the vegetation in the surrounding plains. The data were collected from $900 \mathrm{~m}^{2}$ sampling plots in the woody stratum and $100 \mathrm{~m}^{2}$ plots in the herbaceous stratum, by means of random stratified sampling. We conducted a total of 162 surveys, from which we identified 228 plant species belonging to 43 families and 144 genera. On the inselbergs, identified 184 species in 36 families and 136 genera. Cynanchum hastifolium was the only species identified as strictly endemic to the inselbergs. In the surrounding environment, the flora comprises 194 species belonging to 40 families and 127 genera. The average number of species identified per survey was higher in the areas surrounding the inselbergs $(23.53 \pm 6.28)$ than on the inselbergs themselves (19.93 $\pm 4.83)$. The vegetation on the inselbergs $(719.47 \pm 337.27$ plants/ha) is not as dense as in the surrounding environment $(1,389.91 \pm 674.79$ plants/ha). Given the more pronounced human impacts in the surrounding plains and the relic species and dominant Pterocarpus lucens on the inselbergs, these appear to have become a refuge habitat for certain plant species. The difference between the vegetation on the inselbergs and in the surrounding environment is linked to both edaphic and anthropic factors.

Keywords: inselbergs, phytosociology, ecology, Burkina Faso.

\section{RESUMEN}

\section{VEGETACIÓN DE LOS INSELBERGS DEL SAHEL BURKINÉS}

Los inselbergs son afloramientos rocosos caracterizados por la precariedad de sus factores edáficos y microclimáticos. El objetivo de este estudio es realizar un estudio comparativo entre la vegetación de los inselbergs y la de los ambientes circundantes. Se recopilaron los datos en parcelas de $900 \mathrm{~m}^{2}$ para el estrato leñoso y de $100 \mathrm{~m}^{2}$ para el estrato herbáceo siguiendo un muestreo estratificado y aleatorio. Se realizaron 162 inventarios de vegetación. El estudio permitió inventariar 228 especies vegetales repartidas en 43 familias y 144 géneros. En los inselbergs, se catalogaron 184 especies repartidas en 136 géneros y 36 familias. Sólo la especie Cynanchum hastifolium se catalogó como endémica estricta en los inselbergs. En los ambientes circundantes de los inselbergs, el cortejo florístico se compone de 194 especies repartidas en 40 familias y 127 géneros. El número promedio de especies por inventario es más alto en los ambientes circundantes $(23,53 \pm 6,28)$ que en los inselbergs $(19,93 \pm 4,83)$. La vegetación de los inselbergs $(719,47 \pm$ 337,27 pies/ha) es menos densa que la de los ambientes circundantes (1 389,91 $\pm 674,79 \mathrm{pies} / \mathrm{ha}$ ). El mayor impacto antrópico en las llanuras circundantes, por una parte, y las especies relictas y la dominancia de Pterocarpus lucens en los inselbergs, por otra, serían un reflejo del carácter de refugio que tienen los inselbergs para algunas especies vegetales. La diferencia entre la vegetación de los inselbergs y la de los ambientes circundantes está ligada a factores edáficos y antrópicos.

Palabras clave: inselbergs, fitosociología, ecología, Burkina Faso. 


\section{Introduction}

Les inselbergs sont définis comme des affleurements rocheux, souvent en forme de dôme, qui dominent les pénéplaines avoisinantes, généralement constitués de granite ou de gneiss (Parmentier et al., 2001). Ils constituent des écosystèmes terrestres ressemblant à de véritables îles en raison de leur isolement sur le plan pédologique, climatique et floristique (Müller, 2008).

Les inselbergs présentent des caractéristiques écologiques particulières et se distinguent des milieux environnants. La température de l'air sur les inselbergs de l'Afrique de l'Ouest dépasse $40^{\circ} \mathrm{C}$ et se situe au-dessus de $60^{\circ} \mathrm{C}$ quand elle est mesurée directement sur la roche (Porembski, 2007). Le manque de sol entraîne une faible accumulation d'eau de pluie et l'inclinaison des pentes raides accentue le ruissellement sur les inselbergs. Une étude menée par Poremski et Watve (2005) a montré que le phosphore et l'azote sont sévèrement limités sur les inselbergs. Ces rudes facteurs climatiques et édaphiques des inselbergs différencient leur végétation de celle des plaines environnantes.

Malgré les conditions environnementales extrêmes, les inselbergs constituent des refuges pour de nombreuses espèces végétales dans les régions à forte pression anthropique (Kouassi et al., 2009), comme c'est le cas dans le Sahel burkinabè (Fontès et Guinko, 1995 ; MED, 2006). Les études menées sur la végétation des inselbergs (Muller, 2008 ; Tindano et al., 2011) ont fourni des informations sur la composition floristique et l'état des peuplements ligneux mais n'ont pas mis en exergue la particularité des inselbergs sur le plan floristique vis-à-vis des milieux environnants. Il apparaît donc nécessaire de disposer d'informations sur la particularité des inselbergs sur le plan floristique, par rapport aux plaines environnantes. L'objectif global de cette étude était de comparer la végétation des inselbergs à celle des milieux environnants. Les hypothèses testées étaient les suivantes : la flore des inselbergs se distingue de celle des environs par le nombre d'espèces et la présence d'espèces endémiques ; les peuplements ligneux des inselbergs sont moins anthropisés que ceux des milieux environnants.

Pour ce faire, nous avons cherché, d'une part, à évaluer la différence entre la végétation des inselbergs et celle environnante, à travers une étude phytosociologique et, d'autre part, à préciser le caractère refuge des inselbergs, en étudiant certains paramètres dendrométriques.

\section{Matériel et méthodes Site d'étude}

Le Burkina Faso est situé au cœur de l'Afrique de l'Ouest et s'étend entre la latitude $09^{\circ} 02^{\prime}$ et $15^{\circ} \mathrm{O} 5^{\prime}$ Nord et la longitude $02^{\circ} 02^{\prime}$ Est et $05^{\circ} 03^{\prime}$ Ouest. L'étude a été menée sur les inselbergs de Oursi et de Arbinda, situés dans le secteur phytogéographique nord-sahélien, et ceux de Boussouma, de Kaya, de Kongoussi et de Séguénéga, situés dans le secteur phytogéographique sud-sahélien (figure 1). Le secteur nord-sahélien est caractérisé par une pluviosité moyenne

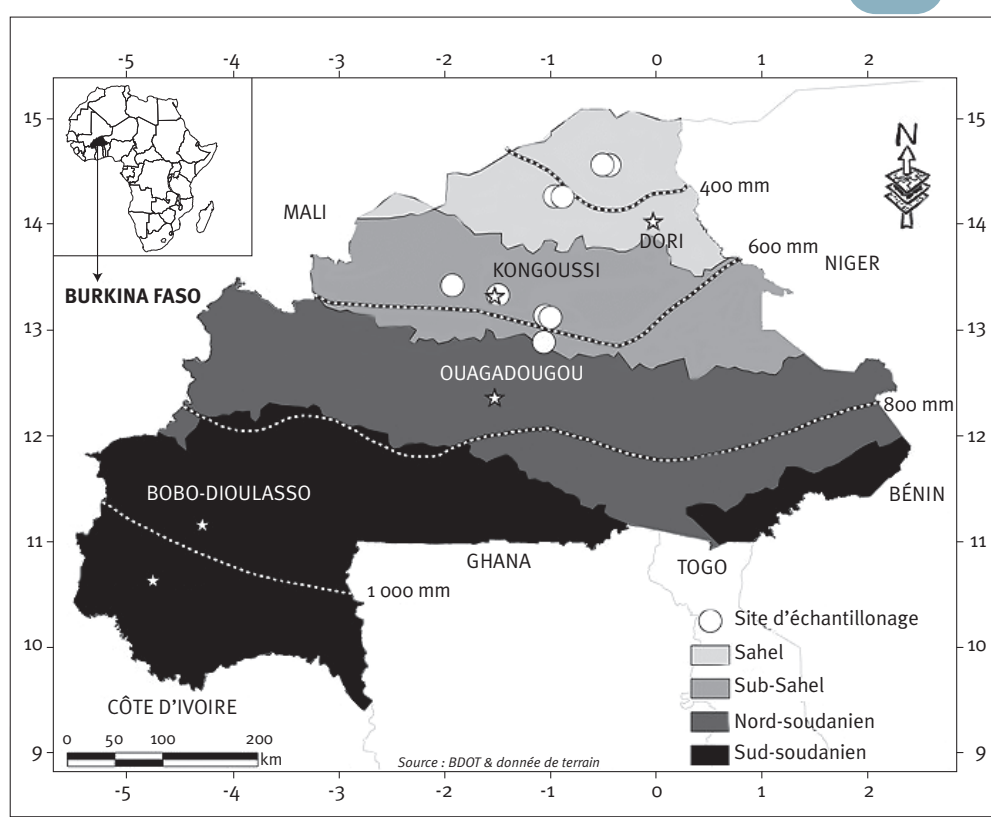

Figure 1.

Localisation des sites d'étude.

annuelle de 400 à 600 mm (Thiombiano et al., 2006). La saison des pluies dure trois à quatre mois. La végétation est dominée par des steppes, des brousses tigrées et des fourrés. Le secteur phytogéographique sud-sahélien est caractérisé par une pluviosité moyenne annuelle de 500 à $700 \mathrm{~mm}$ (Traoré et al., 2012). La saison des pluies dure de trois à cinq mois. La végétation est également dominée par des brousses tigrées et des fourrés.

Les différentes formes d'inselbergs rencontrées dans cette zone sont les affleurements (0,5 à $1 \mathrm{~m}$ ) au-dessus du sol (photo 1), les inselbergs en forme de dôme et enfin les inselbergs tabulaires, qui culminent entre $323 \mathrm{~m}$ et $442 \mathrm{~m}$ (photo 2).

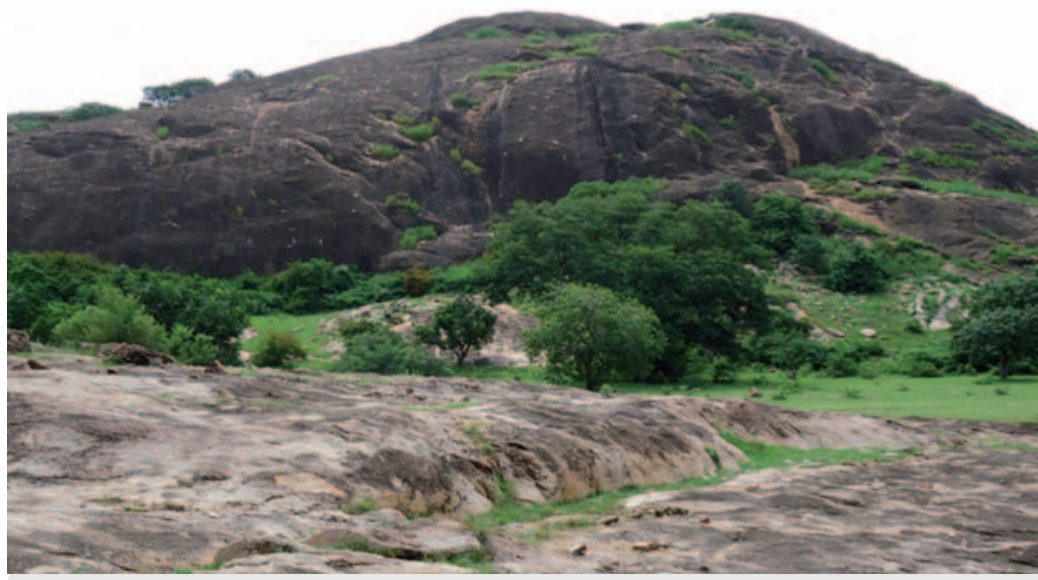

Photo 2.

Inselberg granitique.

Photo E. Tindano, Zongpiiga. 


\section{Collecte des données}

Les données sur les inselbergs ont été collectées suivant un échantillonnage stratifié et aléatoire, représentatif de leur végétation. Les superficies des placeaux étaient de $900 \mathrm{~m}^{2}$ pour la strate ligneuse et $100 \mathrm{~m}^{2}$ pour la strate herbacée. Les données phytosociologiques ont été collectées suivant la méthode sigmatiste de Braun-Blanquet (1932). Pour chaque relevé, la liste exhaustive des espèces végétales affectées de leur coefficient d'abondance-dominance a été dressée. Les missions de terrain se sont déroulées du mois d'août au mois d'octobre des années 2011, 2012, 2013 et 2014, conformément à la période recommandée pour l'inventaire de la flore dans le Sahel burkinabè.

Pour caractériser la végétation ligneuse, les variables renseignées étaient le nombre d'individus de chaque espèce ligneuse et le diamètre du tronc des ligneux ayant un diamètre atteignant au moins $5 \mathrm{~cm}$ (Ouédraogo et Thiombiano, 2012). Le diamètre a été mesuré à $30 \mathrm{~cm}$ du sol en raison de la forte ramification de la plupart des espèces de la zone sahélienne et de la déformation des troncs des plantes due à la pression anthropique (Bognounou, 2009). Pour éviter l'effet de lisière des inselbergs, les relevés des milieux environnants ont été réalisés à une distance d'au moins $500 \mathrm{~m}$ des piedmonts. Dans les placeaux où la roche est couverte par le sol, sa profondeur a été mesurée en trois endroits alignés (Oumorou, 2003).

La vitalité des ligneux a été appréciée au moment du relevé selon les codes suivants : o pour ligneux sans défaut visible ; 1 pour ligneux portant une trace d'exploitation humaine ; 2 pour ligneux partiellement desséché ; 3 pour ligneux totalement desséché ou mort sur pied.

Des placeaux de même dimension que ceux utilisés sur les inselbergs ont été installés dans les milieux environnants pour la collecte des données.

\section{Analyse des données}

\section{Données floristiques}

Les spécimens récoltés ont été identifiés à l'aide de l'ouvrage d'Akoegninou et al. (2006). Les herbiers de l'Université de Ouagadougou et du Centre national de la recherche scientifique et technologique (CNRST) ont servi de références pour la confirmation des noms des espèces. La nomenclature adoptée est celle du catalogue des plantes vasculaires du Burkina Faso (Thiombiano et al., 2012). Les types biologiques et phytogéographiques ont été caractérisés par des spectres bruts et pondérés. Les types biologiques utilisés sont ceux définis par Raunkiaer (1934). Les types phytogéographiques utilisés proviennent des subdivisions chorologiques de White (1986).

La comparaison de la végétation des inselbergs à celle des milieux environnants a été effectuée selon les paramètres suivants :

- l'indice de similitude de Sørensen $C s=2 C /(2 C+B+A)$, $C$ étant le nombre d'espèces communes aux inselbergs et aux milieux environnants, A le nombre d'espèces des inselbergs, et $\mathrm{B}$ le nombre d'espèces des milieux environnants ;

- le nombre moyen d'espèces par placeau $\mathrm{Nm}=\mathrm{Rt} / \mathrm{N}_{\mathrm{r}}$, Rt étant le nombre total d'espèces, et Nr le nombre de relevés; ;
- l'indice de diversité de Shannon $H=\Sigma P_{i} \ln P_{i}$, avec $P_{i}=$ (ni/N), $P_{i}$ étant l'abondance relative de la ième espèce dans un placeau, ni représentant le nombre d'individus de l'espèce $\mathrm{i}$, et $\mathrm{N}$ étant le nombre total d'individus par placeau ;

- l'indice d'équitabilité de Piélou $E=H / \operatorname{lnS}, S$ étant le nombre total des espèces ligneuses.

\section{Données dendrométriques}

Les paramètres suivants ont été calculés pour la caractérisation de la végétation ligneuse :

- la densité $D=N / S$, N étant le nombre total d'individus ligneux, $\mathrm{S}$ la superficie en hectares, D s'exprimant en pieds/ hectare ;

- la surface terrière moyenne $S_{t}=d^{2}{ }_{30 \mathrm{~cm}} \pi / 4, d_{30}$ cm étant le diamètre du tronc à $30 \mathrm{~cm}$ du sol, $\mathrm{S}_{\mathrm{t}}$ s'exprimant en $\mathrm{m}^{2} /$ hectare ;

- la dominance relative

$$
\frac{\text { Surface terrière totale par espèce }}{\text { Surface terrière totale de toutes les espèces }} \times 100
$$

- la densité relative

$$
\frac{\text { Nombre d'individus par espèce }}{\text { Nombre total d'individus de toutes les espèces }} \times 100
$$

- la fréquence

$$
\begin{gathered}
\text { Nombre de relevés où l'espèce est présente } \\
\text { Nombre total des relevés }
\end{gathered}
$$

- la fréquence relative

$\frac{\text { Fréquence par espèce }}{\text { Fréquence totale de toutes les espèces }} \times 100$

- IVI : dominance relative + densité relative + fréquence relative ; les espèces ligneuses dominantes ont été déterminées à partir du calcul des IVI. Toute espèce ligneuse ayant un IVI atteignant au moins $10 \%$ est considérée comme dominante ;

- le taux d'exploitation (Tex) représente le rapport entre le nombre total d'individus portant une trace d'exploitation (coupe, écorçage, déracinement) et l'effectif total du peuplement :

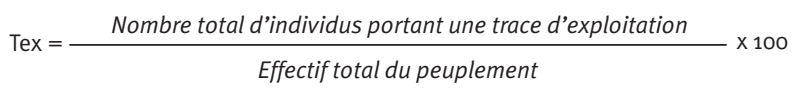

- le taux de dessèchement partiel est le rapport entre l'effectif des individus partiellement desséchés et l'effectif total du peuplement :

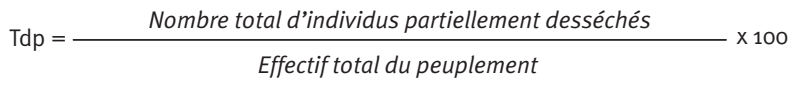

- le taux d'individus morts sur pied est le rapport entre le nombre de pieds morts et l'effectif total du peuplement :

$$
\operatorname{Tmp}=\frac{\text { Nombre de pieds morts }}{\text { Effectif total du peuplement }} \times 100
$$

- le taux de régénération du peuplement est le rapport entre l'effectif total des jeunes plants (individus dont le diamètre du tronc n'atteint pas $5 \mathrm{~cm}$ ) et l'effectif total du peuplement (Ngom et al., 2013) :

$$
\operatorname{Trp}=\frac{\text { Effectif total des jeunes }}{\text { Effectif total du peuplement }} \times 100
$$




\section{Données pédologiques}

La profondeur moyenne du sol des inselbergs a été calculée à partir des différentes mesures selon la formule

$$
\mathrm{Pm}=\frac{\mathrm{P}_{1}+\mathrm{P}_{2}+\mathrm{P}_{3}}{3}
$$

$\mathrm{P}_{1}$ étant la profondeur 1, $\mathrm{P}_{2}$ la profondeur 2, et $\mathrm{P}_{3}$ la profondeur 3.

Les données ne suivant pas une distribution normale, une analyse univariée (Anova de comparaison non paramétrique de Wilcoxon) a été utilisée à l'aide du logiciel JMP o9 pour comparer les moyennes des différents paramètres étudiés.

\section{Résultats}

\section{Composition et diversité floristiques}

Un total de 184 espèces végétales a été recensé sur les inselbergs (tableau I), réparties dans 36 familles et 136 genres (tableau II). Dans les environs des inselbergs, le cortège floristique est composé de 194 espèces réparties en 42 familles et 127 genres. Un ensemble de 34 espèces végétales n'ont été rencontrées que sur les inselbergs (tableau I). À l'inverse, 44 espèces végétales apparaissent inféodées aux milieux environnants.

Sur les inselbergs, le nombre moyen d'espèces par relevé est de 19,93 \pm 4,83. L'indice de diversité de Shannon

\section{Tableau I.}

Liste des espèces végétales recensées sur les inselbergs et les milieux environnants.

\begin{tabular}{|l|c|c|}
\hline Espèces végétales & Inselbergs & $\begin{array}{c}\text { Milieux } \\
\text { environnants }\end{array}$ \\
\hline Acacia ataxacantha DC. & + & + \\
\hline Acacia erythrocalyx Brenan & + & + \\
\hline Acacia laeta R. Br. ex Benth. & + & + \\
\hline Acacia macrostachya Reichenb. ex DC. & + & + \\
\hline Acacia nilotica (L.) Willd. ex Delile & + & + \\
\hline Acacia senegal (L.) Willd. & + & + \\
\hline Acacia seyal Del. & + & + \\
\hline Acacia sieberiana DC. & + & + \\
\hline Acacia tortilis (Forssk.) Hayne & + & + \\
\hline Acalypha ciliata Forssk. & + & + \\
\hline Acanthospermum hispidum DC. & + & + \\
\hline Achyranthes aspera L. & + & + \\
\hline Adansonia digitata L. & + & + \\
\hline Ageratum conyzoides L. & + & + \\
\hline Alysicarpus ovalifolius (Schumach.) J. Léonard & + & + \\
\hline Alysicarpus rugosus (Willd.) DC. & + & + \\
\hline Amaranthus spinosus L. & + & + \\
\hline Amaranthus viridis L. & + & + \\
\hline Ammania senegalensis Lam. & + & + \\
\hline
\end{tabular}

$(H)$, l'indice d'équitabilité de Piélou (E) et l'indice de diversité maximale (LnS) sont respectivement de 2,40 $\pm 0,07,0,63 \pm$ 0,02 et 3,83 (tableau II). Dans les milieux environnants, le nombre moyen d'espèces, l'indice de diversité de Shannon $(H)$, l'équitabilité de Piélou (E) et l'indice de diversité maximale (LnS) sont respectivement de 23,12 \pm 5,48, 2,42 $\pm 0,42,0,70 \pm$ 0,03 et 3,47 (tableau II). L'Anova de Wilcoxon montre que Nm, $\mathrm{H}$ et $\mathrm{E}(\mathrm{p}<0,0003, \mathrm{p}<0,0001$ et $\mathrm{p}<0,0001$ respectivement) diffèrent entre les inselbergs et les milieux environnants.

Les familles dominantes sur les inselbergs sont, par ordre croissant, les Poaceae, les Fabaceae-Faboideae, les Malvaceae et les Fabaceae-Mimosoideae (tableau III). Les types biologiques les plus abondants des inselbergs sont les thérophytes suivis des phanérophytes et des chaméphytes (figure 2). Les types phytogéographiques sont dominés par les espèces soudano-zambéziennes, suivies des espèces des liaisons Afrique tropicale et pantropicale (figure 3).

Les familles qui dominent dans les plaines sont les Poaceae, les Malvaceae, puis les Fabaceae-Faboideae, les Fabaceae-Mimosoideae et les Rubiaceae (tableau III). Les spectres brut et pondéré des types biologiques montrent une dominance des thérophytes, des phanérophytes et des hémicryptophytes (figure 4). Les espèces à distribution soudanozambézienne, pantropicale et Afrique tropicale sont les plus représentées dans les environs des inselbergs (figure 5).

\begin{tabular}{|l|c|c|}
\hline Ampelocissus leonensis (Hook. f.) Planch & - & + \\
\hline Andropogon chinensis (Nees) Merr. & + & - \\
\hline Andropogon fastigiatus Sw. & - & + \\
\hline Andropogon gayanus Kunth. & + & + \\
\hline Anogeissus leiocarpa (DC.) Guill. \& Perr. & + & + \\
\hline Aristida adcensionis L. & + & + \\
\hline Aristida funiculata Trin. \& Rupr. & - & + \\
\hline Aristida hordeacea Kunth & - & + \\
\hline Aristida kerstinguii Pilg. & + & + \\
\hline Aristida mutabilis Trin. \& Rupr. & - & + \\
\hline Aspilia kotschyi (Sch. Bip.) Oliv. & + & + \\
\hline Aspilia rudis Oliv. \& Hiern & + & + \\
\hline Azadirachta indica A. Juss. & + & + \\
\hline Balanites aegyptiaca (L.) Del. & + & + \\
\hline Bauhinia rufescens Lam. & + & + \\
\hline Blepharis linariifolia Pers. & + & + \\
\hline Boerhavia diffusa L. & + & + \\
\hline Bombax costatum Pellegr. et Vuillet. & + & + \\
\hline Boscia angustifolia A. Rich. & + & + \\
\hline Boscia senegalensis (Pers.) Lam. ex Poir. & + & + \\
\hline Boswellia dalzielii Hutch. & + & + \\
\hline Brachiaria lata (Schumach) C.E. Hubb. & + & + \\
\hline Brachiaria villosa (Lam.) A. Camus & + & + \\
\hline Bulbostylis hispidula (Vahl) R. W. Haines & + & + \\
\hline
\end{tabular}




\begin{tabular}{|c|c|c|}
\hline Cadaba glandulosa Forsk. & + & - \\
\hline Calotropis procera (Ait.) Ait. f. & + & + \\
\hline Capparis sepiaria L. var. fisheri (Pax) De Wolf. & + & + \\
\hline Caralluma adscendens (Roxb.) Haw. & + & + \\
\hline Cardiospermum halicacabum L. & + & - \\
\hline Cassia italica (Mill.) Lam. ex F. W. Andrews & - & + \\
\hline Cassia nigricans Vahl & + & - \\
\hline Cassia obtusifolia L. & + & + \\
\hline Cassia occidentalis $\mathrm{L}$. & - & + \\
\hline Cassia sieberiana DC. & + & + \\
\hline Ceiba pentandra (L.) Gaertn & - & + \\
\hline Cenchrus biflorus Roxb. & + & + \\
\hline Cerathoteca sesamoides Endl. & + & + \\
\hline Chamaecrista pratensis (R. Vig.) Du Puy & + & + \\
\hline Chloris pilosa Schumach. & + & + \\
\hline Chloris prieurii Kunth & + & + \\
\hline Chrysanthellum indicum DC. & - & + \\
\hline Cienfuegosia digitata Cav. & + & + \\
\hline Citrullus lanatus (Thunb.) Matsum. \& Nakai Haw. & + & + \\
\hline Cleome monophylla L. & + & + \\
\hline Cleome scaposa DC. & + & + \\
\hline Cleome viscosa $\mathrm{L}$. & + & + \\
\hline Combretum aculeatum Vent. & + & + \\
\hline Combretum glutinosum Perr. ex DC. & + & + \\
\hline Combretum micranthum G. Don. & + & + \\
\hline Combretum nigricans Lepr. ex Guill. \& Perr. & + & + \\
\hline Commelina benghalensis $\mathrm{L}$. & - & + \\
\hline Commelina forskalei Vahl & + & + \\
\hline Commelina subulata Roth & - & + \\
\hline Commiphora africana (A. Rich.) Engl. & + & + \\
\hline Corchorus olitorius Linnaeus & + & + \\
\hline Corchorus tridens $\mathrm{L}$. & + & + \\
\hline Crateva adansonii DC. & - & + \\
\hline Crotalaria goreensis Guill. \& Perr. & + & - \\
\hline Crotalaria retusa $\mathrm{L}$. & + & - \\
\hline Crotalaria senegalensis (Pers.) Bacle ex DC. & + & + \\
\hline Cucumis melo L. [cut.] & + & + \\
\hline Cyanotis lanata Benth. & + & + \\
\hline Cymbopogon schoenanthus (L.) Spreng. & + & + \\
\hline Cynanchum hastifolium K.Schum. & + & - \\
\hline Cyperus esculentus L. [cult.] & + & + \\
\hline Cyperus rotundus L. & - & + \\
\hline Dactyloctenium aegyptium (L.) Willd. & + & + \\
\hline Dalbergia melanoylon Guill. \& Perr. & + & + \\
\hline Desmidorchis acutangula Decne & + & + \\
\hline Desmodium hirtum Guill. \& Perr. & + & + \\
\hline Dichrostachys cinerea (L.) Wight \& Am. & + & + \\
\hline
\end{tabular}

\begin{tabular}{|c|c|c|}
\hline Dicliptera paniculata (Forssk.) I. Darbysh. & + & + \\
\hline Dicoma tomentosa Cass. & + & - \\
\hline Digitaria horizontalis Willd. & + & + \\
\hline Diospyros mespiliformis Hochst. ex A. DC. & + & + \\
\hline Echinochloa colona (L.) Link. & + & + \\
\hline Eleusine indica (L.) Gaertn. & - & + \\
\hline Elionurus elegans Kunth & + & - \\
\hline Eragrostis aspera (lacq.) Nees & + & + \\
\hline Eragrostis cilianensis (All.) Vignolo ex Janch. & - & + \\
\hline Eragrostis tremula Steud. & + & + \\
\hline Eragrostis turgida (Schumach.) De Wild. & - & + \\
\hline Eremomastax polysperma (Benth.) & + & - \\
\hline Euphorbia balsamifera Aiton & + & + \\
\hline Euphorbia convolvuloides Hochstetter ex A. Richard & + & + \\
\hline Euphorbia hirta L. & + & + \\
\hline Evolvulus alsinoides (L.) L. & + & + \\
\hline Faidherbia albida (Delile) A. Chev. & + & + \\
\hline Feretia apodanthera Del. & + & + \\
\hline Ficus sycomorus L. & - & + \\
\hline Fimbristylis ferruginea (L.) Vahl & + & + \\
\hline Flueggea virosa (Roxb. ex Willd.) Voigt. & + & - \\
\hline Gardenia sokotensis Hutch. & + & + \\
\hline Gardenia ternifolia Schumach. et Thonn. & + & + \\
\hline Grewia bicolor Juss. & + & + \\
\hline Grewia flavescens Juss. & + & + \\
\hline Grewia tenax (Forssk.) Fiori & + & + \\
\hline Grewia villosa Willd. & + & + \\
\hline Guiera senegalensis J. G. Gmel. & + & + \\
\hline Gymnosporia senegalensis (Lam.) Loes. & + & + \\
\hline Hackelochloa granularis (L.) Kuntze & + & + \\
\hline Heteropogon contortus (L.) Roem. \& Schult. & - & + \\
\hline Hibiscus calycosus (A. Rich.) & - & + \\
\hline Hibiscus cannabunus L [Cut.] & + & + \\
\hline Hibiscus diversifolius Jacq. & + & + \\
\hline Hyphaene thebaica (L.) Mart. & - & + \\
\hline Indigofera dendroides Jacq. & + & - \\
\hline Indigofera geminata Baker & - & + \\
\hline Indigofera hirsuta L. var. hita & - & + \\
\hline Indigofera pilosa Poir. & + & + \\
\hline Indigofera pulchra Willd. & + & - \\
\hline Indigofera senegalensis Lam. & + & + \\
\hline Indigofera simplicifolia Lam. & + & - \\
\hline Ipomoea asarifolia (Desr.) Roem. \& Schult. & + & + \\
\hline Ipomoea coptica (L.) Roth ex Roem. \& Schult. & + & + \\
\hline Ipomoea coscinosperma Hochst. ex Choisy & + & + \\
\hline Ipomoea dichroa Choisy & - & + \\
\hline Ipomoea eriocarpa R. Br. & + & + \\
\hline
\end{tabular}




\begin{tabular}{|c|c|c|}
\hline Ipomoea involucrata P. Beauv. & + & - \\
\hline Ipomoea quamoclit L. [cult.] & + & + \\
\hline Ipomoea vagans Back. & + & + \\
\hline Khaya senegalensis (Desr.) A. Juss. & - & + \\
\hline Kohautia tenuis (Bowdich) Mabb. & + & + \\
\hline Kyllinga pumila Michx. & - & + \\
\hline Kyllinga squamulata Thonn. ex Vahl & - & + \\
\hline Kyllinga tenuifolia Steud. & - & + \\
\hline Lannea acida A. Rich. & + & + \\
\hline Lannea microcarpa Engl. \& K. Krause & + & + \\
\hline Lannea velutina A. Rich. & + & + \\
\hline Lepidagathis anobrya Nees & + & - \\
\hline Leptadenia hastata (Pers.) Decne. & + & + \\
\hline Leptadenia pyrotechnica (Forssk.) Decne. & + & + \\
\hline Leucas martinicensis (Jacq.) R. Br. & + & + \\
\hline Loudetia togoensis (Pilger) C.E. Hubbard & + & + \\
\hline Ludwigia hyssopifolia (G. Don) Exell & - & + \\
\hline Maerua angolensis DC. & + & + \\
\hline Maerua crassifolia Forsk. & + & + \\
\hline Melantera eliptica 0 . Hoffm. & + & + \\
\hline Merremia pinnata (Hochst. ex Choisy) Hallier f. & + & - \\
\hline Microchloa indica (L. f.) P. Beauv. & + & + \\
\hline Mitracarpus hirtus (L.) DC. & + & + \\
\hline Mitragyna inermis (Willd.) Kuntze & - & + \\
\hline Mollugo nudicaulis Lam. & + & + \\
\hline Ocimum americanum $\mathrm{L}$. & + & + \\
\hline Ocimum irvinei J. K. Morton & + & - \\
\hline Oldenlandia corymbosa L. & - & + \\
\hline Oropetium aristatum (Stapf) Pilg. & + & + \\
\hline Oryza longistaminata A. Chev. \& Roehr. & - & + \\
\hline Pancratium trianthum Herb. & + & + \\
\hline Panicum laetum Kunth & + & + \\
\hline Pennisetum pedicellatum Trin. & + & + \\
\hline Pennisetum polystachion (L.) Schult. & + & + \\
\hline Pentanema indicum (L.) Y. Ling & + & + \\
\hline Pergularia tomentosa $\mathrm{L}$. & + & + \\
\hline Phyllanthus amarus Schumach. \& Thonn. & + & + \\
\hline Phyllanthus maderaspatensis L. & + & + \\
\hline Phyllanthus pentandrus Schumach. \& Thonn. & + & + \\
\hline Physalis angulata $\mathrm{L}$. & + & + \\
\hline Piliostigma reticulatum (DC.) Hochst. & + & + \\
\hline Piliostigma thonningii (Schumach.) Milne-Redh. & + & + \\
\hline Portulaca grandiflora Hook. & - & + \\
\hline Prosopis africana (Guill. et Perr.) Taub. & + & - \\
\hline Pterocarpus erinaceus Poir. & + & + \\
\hline Pterocarpus lucens Lepr. ex Guill. \& Perr. & + & + \\
\hline Pupalea lappacea (L.) A. Juss. & + & + \\
\hline
\end{tabular}

\begin{tabular}{|c|c|c|}
\hline Rhynchosia minima (L.) DC. & + & + \\
\hline Rhynchosia sublobata (Schumach. \& Thonn.) Meikle & + & - \\
\hline Ricinus communis L. & + & - \\
\hline Schizachyrium exile (Hochst.) Pilg. & + & - \\
\hline Schizachyrium sanguineum (Retz.) Alston & + & + \\
\hline Schoenefeldia gracilis Kunth & + & + \\
\hline Sclerocarya birrea (A. Rich.) Hochst & + & + \\
\hline Scoparia dulcis L. & - & + \\
\hline Sesbania pachycarpa DC. & + & + \\
\hline Setaria pumila (Poir.) Roem. et Schult. & + & + \\
\hline Sida acuta Burm. f. & + & + \\
\hline Sida alba L. & + & + \\
\hline Sida cordifolia L. & + & - \\
\hline Sida ovata Forssk. & + & - \\
\hline Sida rhombifolia L. & + & - \\
\hline Spermacoce chaetocephala de Candolle & + & + \\
\hline $\begin{array}{l}\text { Spermacoce filifolia (Schumach. \& Thonn.) } \\
\text { J.-P. Lebrun \&Stok }\end{array}$ & - & + \\
\hline Spermacoce radiata (DC.) Sieber ex Hiern & + & + \\
\hline Spermacoce stachydea DC. & + & + \\
\hline Sporobolus festivus Hochst. ex A. Rich. & + & + \\
\hline Stachytarpheta angustifolia (Miller) Vahl & - & + \\
\hline Sterculia setigera Del. & + & + \\
\hline Sterospermum kunthianum Cham. & + & + \\
\hline Striga hermontheca (Del.) Benth. & - & + \\
\hline Stylochaeton hypogaeus Lepr. & - & + \\
\hline Stylosantes erecta P. Beauv. & + & - \\
\hline Tamarindus indica $\mathrm{L}$. & + & + \\
\hline Tephrosia bracteolata Guill. \& Perr. & + & + \\
\hline Tephrosia linearis (Willd.) Pers. & + & + \\
\hline Tephrosia nana Schweinf. & + & - \\
\hline Tephrosia pedicellata Baker & + & - \\
\hline Tetrapogon cenchriformis (A. Rich.) Clayton & + & + \\
\hline Tinospora bakis (A. Rich.) Miers & + & + \\
\hline Tragus berteronianus Schult. & - & + \\
\hline Tragus racemosus (L.) All. & + & - \\
\hline Tribulus terrestris Linnaeus & + & + \\
\hline Tripogon minimus (A. Rich.) Steud. & - & + \\
\hline Triumfetta pentandra A. Rich. & - & + \\
\hline Vitellaria paradoxa Gaertn. f. & + & + \\
\hline Walteria indica $\mathrm{L}$. & + & + \\
\hline Wissadula amplissima (L.) R.E. & + & + \\
\hline Ximenia americana $\mathrm{L}$. & + & + \\
\hline Ziziphus mauritiana Lam. & + & + \\
\hline Zornia glochidiata Rchb. ex DC. & + & + \\
\hline
\end{tabular}

+ : présence ; - : absence. 
Tableau II.

Diversité floristique des inselbergs et des milieux environnants (moyenne \pm écart-type).

\begin{tabular}{|l|l|c|c|c|c|c|c|c|}
\hline Familles & Genres & Espèces & Nm & H & E & LnS & Cs & \\
\hline Inselbergs & Nord-sahélien & 30 & 88 & 123 & $20,10 \pm 4,25$ & $2,39 \pm 0,09$ & $0,66 \pm 0,02$ & 3,61 \\
\hline & Sud-sahélien & 32 & 90 & 128 & $20,49 \pm 5,59$ & $1,96 \pm 0,06$ & $0,50 \pm 0,02$ & 3,93 \\
Milieux \\
environnants
\end{tabular}

$\mathrm{Nm}$ : nombre moyen d'espèces; $\mathrm{H}$ : indice de diversité de Shannon ; $\mathrm{E}$ : indice d'équitabilité de Piélou ; LnS : indice de diversité maximale ; $\mathrm{s}$ : indice de similitude de Sorensen.

Tableau III.

Spectre brut des familles.

\begin{tabular}{|c|c|c|c|c|c|c|}
\hline \multirow[b]{3}{*}{ Familles } & \multicolumn{6}{|c|}{ Spectre brut (\%) } \\
\hline & \multicolumn{2}{|c|}{ Inselbergs } & \multirow[b]{2}{*}{ Total } & \multicolumn{3}{|c|}{ Milieux environnants } \\
\hline & Nord-Sahel & Sud-Sahel & & Nord-Sahel & Sud-Sahel & Total \\
\hline Acanthaceae & 2,44 & 2,34 & 2,39 & 0,89 & 0,73 & 0,80 \\
\hline Amaranthaceae & 2,44 & 1,56 & 1,99 & 2,68 & 2,19 & 2,41 \\
\hline Amaryllidaceae & 0,81 & - & 0,40 & 0,89 & - & 0,40 \\
\hline Anacardiaceae & 1,63 & 3,13 & 2,39 & - & 2,19 & 1,20 \\
\hline Apocynaceae & 5,69 & 1,56 & 3,59 & 2,68 & 2,19 & 2,41 \\
\hline Arecaceae & - & - & - & - & 0,73 & 0,40 \\
\hline Asteraceae & 2,44 & 3,91 & 3,19 & 0,89 & 0,73 & 0,40 \\
\hline Bignoniaceae & - & 0,78 & 0,40 & - & 0,73 & 0,40 \\
\hline Burseraceae & 0,81 & 1,56 & 1,20 & 0,89 & 4,38 & 0,40 \\
\hline Capparaceae & 6,50 & 4,69 & 5,58 & 6,25 & 4,38 & 5,22 \\
\hline Combretaceae & 3,25 & 4,69 & 3,98 & 4,46 & 2,92 & 4,42 \\
\hline Commelinaceae & 0,81 & 1,56 & 1,20 & 0,89 & 1,46 & 2,01 \\
\hline Convolvulaceae & 6,50 & 1,56 & 3,98 & 7,14 & 1,46 & 4,02 \\
\hline Cucurbitaceae & 1,63 & 0,78 & 1,20 & 1,79 & 5,11 & 1,61 \\
\hline Cyperaceae & 1,63 & 1,56 & 1,59 & 0,89 & 0,73 & 3,21 \\
\hline Ebenaceae & - & 0,78 & 0,40 & - & 0,73 & 0,40 \\
\hline Euphorbiaceae & 1,63 & 1,56 & 1,59 & 0,89 & 5,11 & 0,40 \\
\hline Fabaceae-Caesalpinoideae & 3,25 & 7,03 & 5,18 & 4,46 & 7,30 & 4,82 \\
\hline Fabaceae-Faboideae & 11,38 & 10,16 & 10,76 & 8,93 & 5,84 & 8,03 \\
\hline Fabaceae-Mimosoideae & 4,07 & 9,38 & 6,77 & 5,36 & 0,73 & 5,62 \\
\hline Lamiaceae & 0,81 & 0,78 & 0,80 & - & 0,73 & 0,40 \\
\hline Lythraceae & 0,81 & - & 0,40 & - & - & - \\
\hline Malvaceae & 11,38 & 8,59 & 9,96 & 10,71 & 10,95 & 10,84 \\
\hline Menispermaceae & 0,81 & - & 0,40 & - & - & - \\
\hline Meliaceae & - & 0,78 & 0,40 & - & 1,46 & 0,80 \\
\hline Molluginaceae & 0,81 & 0,78 & 0,80 & 0,89 & 0,73 & 0,80 \\
\hline Moraceae & - & - & - & - & 0,73 & 0,40 \\
\hline
\end{tabular}




\begin{tabular}{|l|c|c|c|c|c|c|}
\hline Nyctaginaceae & 0,81 & 0,78 & 0,80 & 0,89 & 0,73 & 0,80 \\
\hline Onagraceae & - & - & - & 0,89 & 1,46 & 0,80 \\
\hline Pedaliaceae & 0,81 & 0,78 & 0,80 & 0,89 & 20,44 & 0,80 \\
\hline Phyllanthaceae & 1,63 & 2,34 & 1,99 & 1,79 & 0,73 & 1,61 \\
\hline Plantaginaceae & - & - & - & - & 0,73 & 0,40 \\
\hline Poaceae & 18,70 & 17,19 & 17,93 & 24,11 & 6,57 & 22,09 \\
\hline Portulacaceae & - & - & - & - & 0,73 & 0,40 \\
\hline Rhamnaceae & 0,81 & 0,78 & 0,80 & 0,89 & 0,73 & 0,80 \\
\hline Rubiaceae & 3,25 & 5,47 & 4,38 & 4,46 & - & 5,62 \\
\hline Sapotaceae & - & 0,78 & 0,40 & - & 0,73 & 0,40 \\
\hline Scrophulariaceae & - & - & - & 0,89 & - & 0,40 \\
\hline Solanaceae & - & 0,78 & - & - & 0,73 & 0,40 \\
\hline Sapindaceae & 0,81 & - & 0,40 & - & - & - \\
\hline Vitaceae & - & - & - & 0,89 & 0,73 & 0,40 \\
\hline Ximeniaceae & 1,63 & 0,78 & 1,20 & 1,79 & - & 0,40 \\
\hline Zygophyllaceae & & 0,78 & 0,40 & - & - & 1,20 \\
\hline
\end{tabular}

- : absence.

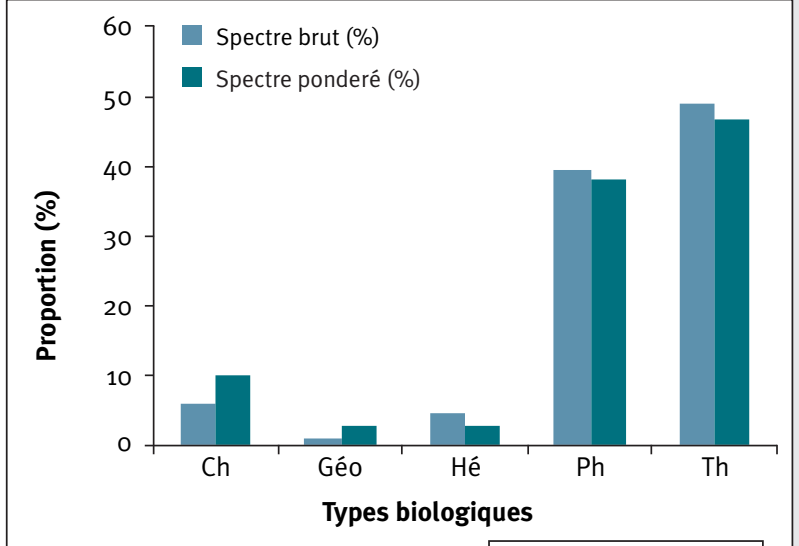

Figure 2.

Types biologiques des inselbergs.

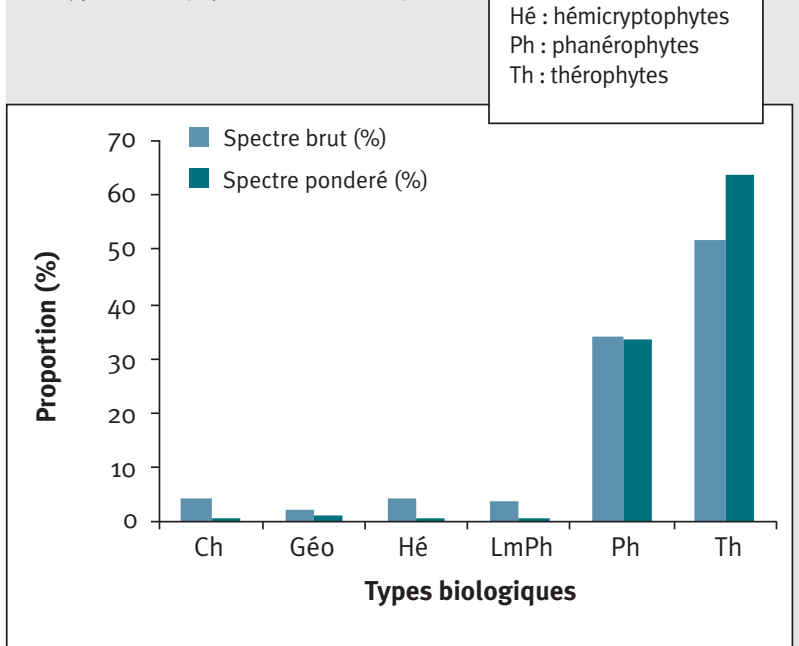

Figure 4.

Types biologiques des milieux environnants.

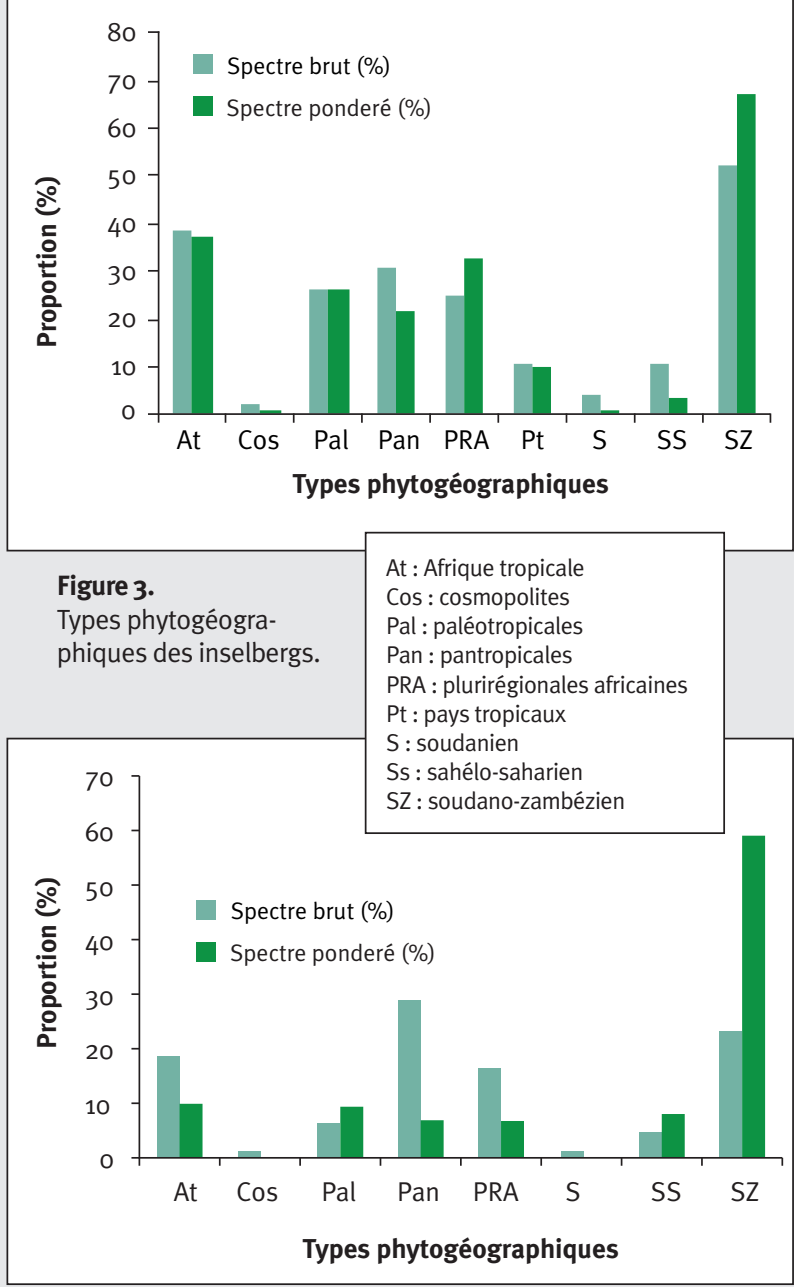

Figure 5.

Types phytogéographiques des milieux environnants. 


\section{Caractéristiques des peuplements ligneux}

La densité moyenne, le diamètre moyen et la surface terrière moyenne calculés pour la végétation ligneuse des inselbergs et celle des milieux environnants sont consignés dans le tableau IV. La densité moyenne, le diamètre moyen et la surface terrière moyenne diffèrent significativement entre les inselbergs et les milieux environnants $(p<0,0001)$. Les plus faibles taux de dessèchement partiel $(8,96 \%)$ et de mortalité (2,76\%) des ligneux, et le fort taux d'exploitation $(12,32 \%)$ s'observent dans les environs des inselbergs. Les plus forts taux de dessèchement partiel $(12,52 \%)$ et de mortalité $(4,23 \%)$, et le plus faible taux d'exploitation des ligneux (8,6 \%) sont relevés sur les inselbergs (figure 6).

Les espèces ligneuses dominantes des inselbergs sont Guiera senegalensis, Commiphora africana, Pterocarpus lucens et Combretum micranthum (tableau V). Dans les

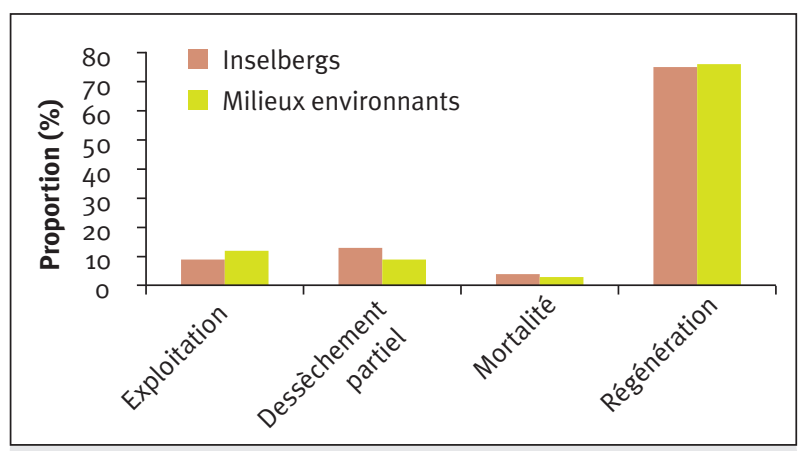

Figure 6.

Vitalité et renouvellement des peuplements ligneux.

plaines, les espèces dominantes sont Balanites aegyptiaca (52,84\%), Acacia tortilis (41,62\%) et Acacia nilotica (23,41\%) (tableau V).

Tableau IV.

Moyenne des paramètres dendrométriques de la végétation ligneuse des inselbergs et des milieux environnants.

\begin{tabular}{|l|l|c|c|c|c|}
\hline & & $\begin{array}{c}\text { Densité moyenne } \\
(\text { pieds/ha) }\end{array}$ & $\begin{array}{c}\text { Diamètre moyen } \\
(\mathbf{c m})\end{array}$ & $\begin{array}{c}\text { Hauteur moyenne } \\
(\mathrm{m})\end{array}$ & $\begin{array}{c}\text { Surface terrière moyenne } \\
\left(\mathrm{m}^{2} / \mathrm{ha}\right)\end{array}$ \\
\hline \multirow{4}{*}{ Inselbergs } & Nord-sahélien & $729,31 \pm 449,64$ & $10,23 \pm 5,20$ & $2,48 \pm 2,27$ & $25,60 \pm 18,87$ \\
\cline { 2 - 6 } & Sud-sahélien & $709,63 \pm 224,90$ & $11,67 \pm 8,27$ & $2,64 \pm 1,53$ & $15,77 \pm 12,51$ \\
\cline { 2 - 6 } & Total & $719,47 \pm 337,27$ & $10,60 \pm 6,18$ & $2,52 \pm 2,09$ & $20,68 \pm 6,95$ \\
\hline \multirow{2}{*}{$\begin{array}{l}\text { Milieux } \\
\text { environnants }\end{array}$} & Nord-sahélien & $2070,18 \pm 1124,67$ & $13,36 \pm 8,91$ & $1,48 \pm 1,35$ & $109,94 \pm 76,42$ \\
\cline { 2 - 6 } & Sud-sahélien & $709,63 \pm 224,90$ & $11,81 \pm 9,78$ & $1,44 \pm 1,41$ & $31,34 \pm 22,27$ \\
\cline { 2 - 6 } & Total & $1389,91 \pm 674,79$ & $12,59 \pm 9,35$ & $1,46 \pm 1,38$ & $70,64 \pm 38,29$ \\
\hline
\end{tabular}

Tableau V.

Indice de valeurs d'importance (IVI) des espèces dominantes des inselbergs et des milieux environnants.

\begin{tabular}{|l|c|c|c|c|c|c|}
\hline & \multicolumn{5}{|c|}{ IVI (\%) } \\
\hline Espèces & Nord-Sahel & Sud-Sahel & Total & Nord-Sahel & Sud-Sahel & Total \\
\hline Acacia laeta & 27,52 & 4,82 & 13,46 & 14,94 & - & 10,61 \\
\hline Acacia nilotica & 5,30 & 2,30 & 3,54 & 25,08 & 7,21 & 23,41 \\
\hline Acacia tortilis & 17,47 & 1,66 & 13,39 & 70,24 & - & 41,62 \\
\hline Acacia seyal & 0,31 & 33,27 & 11,10 & 27,07 & 27,76 & 19,65 \\
\hline Anogeissus leiocarpa & - & 13,20 & 5,29 & 14,68 & 1,23 & 13,08 \\
\hline Balanites aegyptiaca & 13,52 & 11,62 & 10,72 & 58,10 & 55,68 & 52,84 \\
\hline Combretum aculeatum & 9,82 & - & 1,15 & 10,35 & 7,64 & 13,93 \\
\hline Combretum glutinosum & 3,04 & 6,39 & 3,53 & 5,66 & 12,94 & 10,58 \\
\hline Combretum micranthum & 12,91 & 31,17 & 17,69 & 7,79 & 22,08 \\
\hline Commiphora africana & 66,29 & 3,53 & 42,15 & 0,96 & 22,20 \\
\hline Guiera senegalensis & 26,31 & 72,33 & 60,17 & 8,63 & - & 0,82 \\
\hline Lannea micocapa & 0,91 & 10,85 & 3,53 & - & 17,37 \\
\hline Leptadenia hastata & 10,88 & - & 0,63 & 11,50 & 11,36 \\
\hline Piliostigma reticulatum & 2,74 & 6,28 & 8,71 & 4,45 & 10,43 & 10,87 \\
\hline Pterocarpus lucens & 42,04 & - & 30,29 & 13,62 & 39,03 & 11,60 \\
\hline Ziziphus mauritiana & 7,71 & 2,94 & 10,40 & 12,13 & - \\
\hline
\end{tabular}




\section{Discussion}

Selon Adjanohoun (1964), les inselbergs se caractérisent par la présence d'espèces telles que Ophioglossum costatum, Ophioglossum thomasii, Drosera indica, Xyris anceps, Xyris capensis. Afrotrilepis pilosa et Cyanotis lanata figurent parmi les espèces endémiques des inselbergs dénombrées par Porembski (2000). Lebrun et al. (1991) ont révélé que Cynanchum hastifolium était une espèce caractéristique des inselbergs (photo 3).

Comparativement à ces études, les inselbergs du Sahel burkinabè se caractérisent au contraire par l'absence d'espèces caractéristiques. Seules Cynanchum hastifolium et Cyanotis lanata ont été recensées comme les deux espèces endémiques sur ces inselbergs. L'absence la plus marquante est celle de Afrotrilepis pilosa, spécialiste stricte des inselbergs selon Kouassi et al. (2014). Cette absence d'espèces endémiques des inselbergs dans le Sahel burkinabè semble s'expliquer par la position latitudinale de notre zone d'étude. En effet, les espèces endémiques des inselbergs comme Lindernia exilis, Afrotrilepis pilosa et Dopatrium longidens se rencontrent sur les inselbergs du domaine soudanien du Burkina Faso (Ouédraogo et Schmidt, 2010).

Les familles dominantes des inselbergs du Sahel burkinabè, comparativement à celles révélées par des études menées en zone soudanienne, suggèrent de manière similaire que l'absence de certaines familles caractéristiques des inselbergs est ici due à la position latitudinale. Sur les inselbergs de la Côte d'Ivoire et du Gabon, les familles dominantes sont les Rubiaceae, les Cyperaceae, les Orchidaceae, les Euphorbiaceae, les Fabaceae-Caesalpinoideae et les Poaceae (Bonardi, 1966 ; Kouassi et al., 2009 ; N'gok Banak, 2005). Dans la présente étude, ce sont en revanche plutôt les Poaceae, les Fabaceae-Faboideae, les Malvaceae et les Fabaceae-Mimosoideae qui dominent. Parmentier (2005) a révélé que les inselbergs des zones humides se distinguent de ceux des zones sèches par l'abondance des Orchidaceae et des ptéridophytes. Nos résultats corroborent ceux de Oumorou et Lejoly (2003) pour la dominance des Poaceae et des Fabaceae-Faboideae.

La différence entre les inselbergs et leurs environs repose sur leur richesse floristique et les espèces propres à chacune de ces zones. En termes de richesse spécifique, les milieux environnants abritent plus d'espèces végétales (194) que les inselbergs (184). La flore des milieux environnants se caractérise par des espèces anthropiques (Commelina benghalensis, Chrysanthellum indicum, Amaranthus viridis, Aristida hordeacea, Acalypha ciliata, Andropogon fastigiatus, Striga hermontheca, Kylliga squamulata, Eleusine indica, Cassia occidentalis, Triumfetta pentandra) et de zones perturbées (Scoparia dulcis, Ipomoea dichroa, Indigofera geminata) (Akoegninou et al., 2006), qui sont absentes sur les inselbergs. Cette absence indiquerait l'état naturel de la végétation des inselbergs par rapport à celle des plaines. Les inselbergs se caractérisent quant à eux par des espèces endémiques telles que Cynanchum hastifolium, Cyanotis lanata selon Lebrun et al. (1991) et Porembski (2000). Cynanchum hastifolium habite seulement les rochers et constitue de ce fait une espèce endémique stricte de ces milieux. La rareté des individus de cette espèce, même sur les inselbergs, et son absence totale dans les plaines font d'elle une espèce rare au Burkina Faso. À ces espèces s'ajoutent les espèces reliques sur inselbergs (Prosopis africana, Lepidagathis anobrya, Cadaba glandulosa, Cardiospermum halicacabum, Boswellia dalziellii, Blepharis linearifolia, Andropogon chinensis) qui sont devenues très rares, ou ont même disparu des plaines. Le nombre moyen d'espèces par relevé, les indices de diversité maximale et de Shannon prouvent que la végétation des milieux environnants est plus riche et plus diversifiée en espèces que celle des inselbergs. Mais le coefficient de similarité de Sorensen (Cs $=79 \%$ ) indique une similitude entre les flores de ces zones. Les types biologiques et phytogéographiques dominants sont les mêmes pour les deux zones. La végétation ligneuse des inselbergs est moins dense que celle des environs (729,31 pieds/ha contre 1389,91 pieds/ha). Elle présente le plus faible diamètre moyen et la plus faible surface terrière moyenne par rapport à la végétation environnante.

La différence entre la végétation des inselbergs et celle des environs s'explique par les conditions de vie sévères que rencontre la végétation des inselbergs, conditions qui, dans le domaine sahélien, sont marquées par l'absence ou la faible profondeur du sol, entraînant une extrême aridité. En effet, la profondeur moyenne du sol est de 5,01 $\pm 2,70 \mathrm{~cm}$ sur les inselbergs. Oumorou (2003) a montré que le facteur déterminant sur les inselbergs est la sécheresse édaphique, due à l'absence de réserves hydriques. Porembski (2007) a mentionné également la forte insolation et la hausse des températures sur les inselbergs. La végétation des milieux environnants bénéficie d'un sol plus profond et plus humide. La profondeur du sol constitue donc une donnée écologique de première importance sur les inselbergs (Oumorou et Lejoly, 2003). Elle y joue le rôle de facteur limitant et détermine l'existence et la répartition des formations végétales. Les forts taux de dessèchement partiel et de mortalité

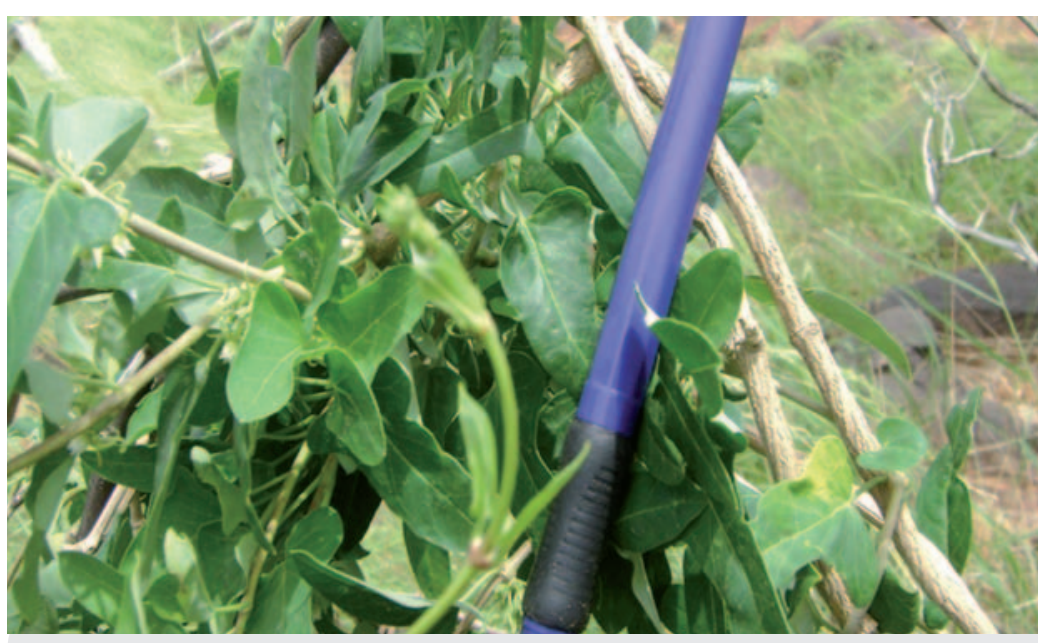

Photo 3.

Cynanchum hastifolium K. Schum., espèce confinée aux inselbergs. Photo E. Tindano, Kollel. 
obtenus dans notre étude confirment les conditions écologiques drastiques auxquelles est soumise la végétation des inselbergs. Cependant, malgré leurs conditions écologiques sévères, les inselbergs constituent des zones refuges pour certaines espèces telles que les espèces reliques citées ci-dessus, face à la pression anthropique. Par ailleurs, la dominance de Pterocarpus lucens sur les inselbergs témoigne de leur caractère refuge pour cette espèce qui fait partie des espèces menacées du domaine sahélien (Thiombiano et Kampmann, 2010), mais la menace est beaucoup plus accentuée dans les environs que sur les inselbergs.

Le caractère refuge des inselbergs s'illustre également par le faible taux d'exploitation (8,06 \%) qu'on y enregistre par rapport à celui observé dans les milieux environnants $(12,32 \%)$. Le faible taux d'exploitation s'expliquerait par les conditions d'accès difficiles pour les inselbergs. L'anthropisation de la végétation des inselbergs se limite à quelques coupes de bois et à un pâturage sporadique (photo 4), comme l'ont indiqué Wittig et al. (2000). La végétation des milieux environnants est soumise à une anthropisation intensive (photo 5) dont la composante majeure est l'exploitation agricole. Les environs de certains inselbergs sont en effet envahis par des champs (photo 6).

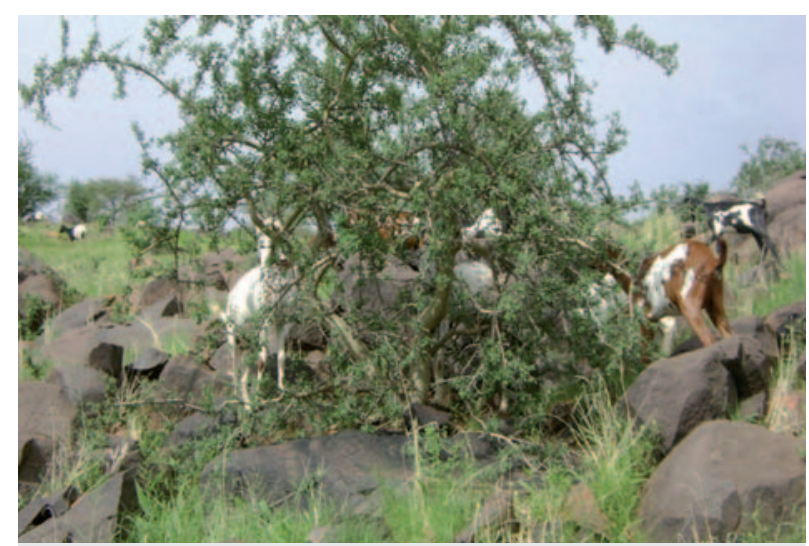

Photo 4.

Inselberg investi par des caprins.

Photo E. Tindano, Kollel.

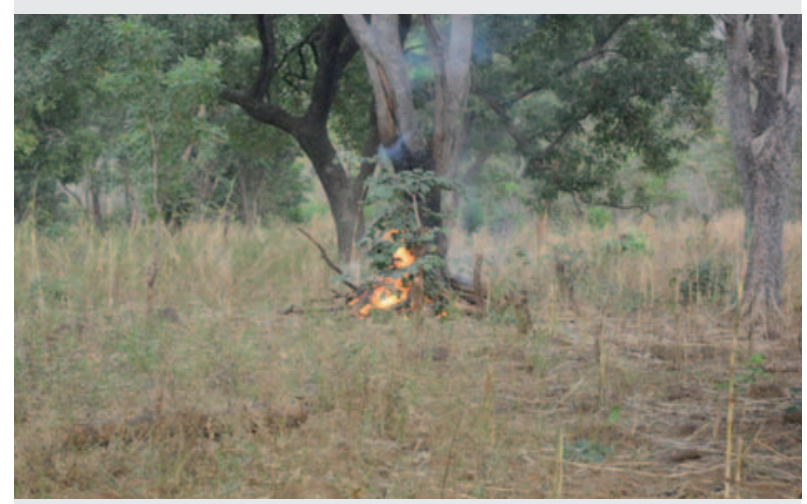

Photo 5.

Défrichage de ligneux par le feu dans un champ de mil. Photo E. Tindano, Tiara.

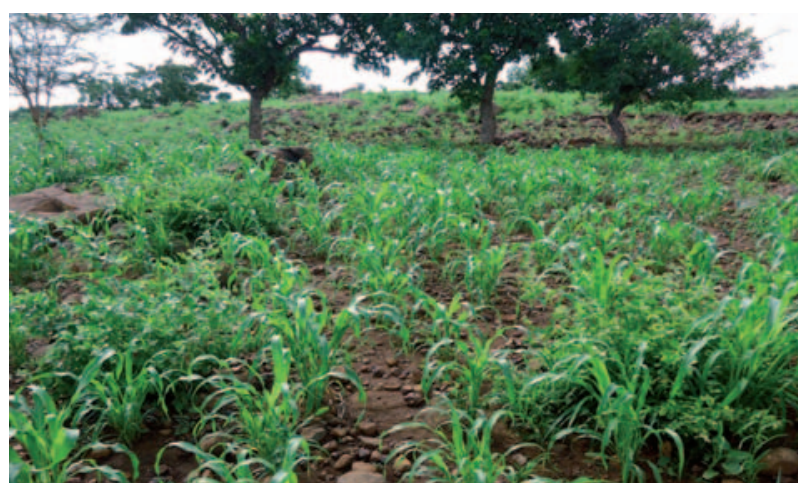

Photo 6.

Champs de sorgho au piedmont d'un inselberg.

Photo E. Tindano, Boussouma.

\section{Conclusion}

Cette étude nous a permis de caractériser la végétation des inselbergs par rapport à celle des plaines. Au total, 184 espèces réparties dans 36 familles et 136 genres ont été recensées sur les inselbergs. Parallèlement, 194 espèces réparties en 42 familles et 127 genres ont été recensées dans les plaines. Au cours de cette étude, une seule espèce spécialiste stricte des inselbergs (Cynanchum hastifolium) a été observée, ce qui montre que la plupart des espèces des inselbergs peuvent vivre dans les plaines. La végétation des inselbergs se distingue de celle des plaines environnantes par sa faible richesse floristique, sa faible densité, les forts taux de dessèchement partiel et de mortalité de la végétation ligneuse. La végétation des milieux environnants est plus dense et plus riche en espèces, mais elle est sous le joug d'une anthropisation galopante. Le principal facteur écologique qui explique la différence entre la végétation des inselbergs et celle des environs est le facteur édaphique. La supériorité du nombre d'espèces recensées dans les plaines et la présence de Cynanchum hastifolium sur les inselbergs confirment notre première hypothèse. Le faible taux d'exploitation enregistré, l'existence d'espèces reliques et la dominance de Pterocarpus lucens sur les inselbergs valident la seconde.

\section{Références bibliographiques}

Adjanohoun E., 1964. Végétation des savanes et des rochers découverts en Côte d'ivoire Centrale. Paris, France, Orstom (IRD), Mémoire $\mathrm{n}^{\circ} 7,238 \mathrm{p}$.

Akoegninou A., Van Der Burg W. J., Van Der Maesen L. J. G., 2006. Flore analytique du Bénin. Wageningen, Pays-Bas, Backhuys Publishers, 1034 p.

Bognounou F., 2009. Restauration écologique et gradient latitudinal : utilisation, diversité et régénération de cinq espèces de Combretaceae au Burkina Faso. Thèse de doctorat, Université de Ouagadougou, Unité de recherche et de formation en Sciences de la Vie et de la Terre, Burkina Faso, 179 p. 
Bonardi D., 1966. Contribution à l'étude botanique des inselbergs de Côte d'Ivoire forestière. Diplôme d'études supérieures en sciences biologiques, Université d'Abidjan, Côte d'Ivoire, $81 \mathrm{p}$.

Braun-Blanquet J., 1932. Plant sociology. The study of plant communities. New York, Londres, McGray Hill, 439 p.

Fontès J., Guinko S., 1995. Carte de la végétation et de l'occupation du sol du Burkina Faso. Université de Ouagadougou, Burkina Faso, 53 p.

Kouassi R. H., Tiebre M.-S., N’guessan K. E., 2009. Aperçu de la végétation des Inselbergs Brafouéby et Mafa-Mafou (Sud-Est de la Côte d'Ivoire). European Journal of Scientific Research, $28:$ 92-123.

Kouassi R. H., Tiebre M.-S., Kouassi K. H., N'guessan K. E., 2014. Diversité floristique des inselbergs Brafouéby et MafaMafou (Sud-Est de la Côte d'Ivoire). Journal of Animal and Plant Sciences, 22 (1) : 3407-3418.

Lebrun J.-P., Toutain B., Gaston A., Boudet G., 1991. Catalogue des plantes vasculaires du Burkina Faso. Maisons-Alfort, France, Cirad-IEMVT, Études et synthèses de l'IEMVT, $n^{\circ}$ 40, $341 \mathrm{p}$.

Ministère de l'Économie et du Développement (MED), 2006. Atlas du Burkina Faso, 215 p.

Müller J., 2008. Herbaceous and non-inundated vegetation of Sahelian inselbergs in Burkina Faso. Candollea, 63: 57-79.

N'gok Banak L., 2005. Diversité végétale des inselbergs et des dalles rocheuses du Nord Gabon. Thèse de doctorat, Université libre de Bruxelles, Belgique, $419 \mathrm{p}$.

Ngom D., Fall T., Sarr O., Diatta S., Akpo L. E., 2013. Caractéristiques écologiques du peuplement ligneux de la réserve de biosphère du Ferlo (Nord Sénégal). Journal of Applied Biosciences, 65 : 5008-5023.

Ouédraogo O., Schmidt M., 2010. Chaînes gréseuses et formations saxicoles. In : Thiombiano A., Kampmann D. (éds). Atlas de la biodiversité de l'Afrique de l'Ouest. Tome II : Burkina Faso. Ouagadougou et Francfort-sur-le-Main, BIOTA, $592 \mathrm{p}$.

Ouédraogo A., Thiombiano A., 2012. Regeneration pattern of four threatened tree species in Sudanian savannas of Burkina Faso. Agroforestry Systems, 861: 35-48.

Oumorou M., 2003. Étude écologique, floristique, phytogéographique et phytosociologique des inselbergs du Bénin. Thèse de doctorat, Université libre de Bruxelles, Belgique, $231 \mathrm{p}$.

Oumorou M., Lejoly J., 2003. Écologie, flore et végétation de l'inselberg Sobakpérou (Nord-Bénin). Acta Botanica Gallica, $150: 65-84$.
Parmentier I., 2005. Étude de la végétation des inselbergs de la forêt dense d'Afrique centrale atlantique. Acta Botanica Gallica, 152 : 403-408.

Parmentier I., Lejoly J., Nguema N., 2001. La végétation des inselbergs de Piedra Nzas (Guinée Équatoriale continentale). Acta Botanica Gallica, 148 : 341-365.

Porembski S., 2000. The invasibility of tropical granite outcrops ("inselbergs") by exotic weeds. Journal of the Royal Society of Western Australia, 83: 131-137.

Porembski S., 2007. Effects of anthropogenic disturbance on the vegetation of granitic and gneissic rock outcrops (inselberg) in West Africa. Nova Hedwigia, Beiheft, 131: 237-246.

Porembski S., Watve A., 2005. Remarks on the species composition of ephemeral flush communities on paleotropical rock outcrops. Phytocoenologia, 35 (2-3): 389-402.

Raunkiaer C., 1934. The life forms of plants and statistical plant geography. Oxford, Royaume-Uni, Clarendon Press, $632 \mathrm{p}$.

Thiombiano A., Schmidt M., Kreft H., Guinko S., 2006. Influence du gradient climatique sur la distribution des espèces Combretaceae au Burkina Faso (Afrique de l'Ouest). Candollea, 61: 189-213.

Thiombiano A., Kampmann D. (éds), 2010. Atlas de la biodiversité de l'Afrique de l'Ouest. Tome II : Burkina Faso. Ouagadougou et Francfort-sur-le-Main, BIOTA, 592 p.

Thiombiano A., Schmidt M., Dressler S., Ouédraogo A., Hahn K., Zizka G., 2012. Catalogue des plantes vasculaires du Burkina Faso. Genève, Suisse, Conservatoire et Jardin botaniques de la Ville de Genève, Boissiera, 65, 391 p.

Tindano E., Ganaba S., Thiombiano A., 2011. Rocky woody vegetation diversity and structure in the Oursi dam area, Northern Burkina Faso. ISESCO Journal of Science and Technology, 7 (12): 15-28.

Traoré S., Zerbo L., Schmidt M., Thiombiano L., 2012. Acacia communities and species responses to soil and climate gradients in the Sudano-Sahelian zone of West Africa. Journal of Arid Environments, 87: 144-152.

White F., 1986. La végétation d'Afrique. Mémoire accompagnant la carte de la végétation de l'Afrique. Unesco/AETFAT/ UNSO, Orstom-Unesco, $384 \mathrm{p}$.

Wittig R., Hahn-Hadjali K., Thiombiano A., 2000. Les particularités de la végétation et de la flore de la chaîne du Gobnangou dans le Sud-Est du Burkina Faso. Études sur la flore et la végétation du Burkina Faso et des pays avoisinants, 5: 49-64. 